OPEN ACCESS

Edited by:

Isabelle Bekeredjian-Ding, Paul-Ehrlich-Institut (PEI), Germany

Reviewed by:

Karen Huber,

Paul-Ehrlich-Institut (PEI), Germany

Roland Lang,

University Hospital Erlangen, Germany

${ }^{*}$ Correspondence:

Manuel Fresno

mfresno@cbm.csic.es

${ }^{\dagger}$ Deceased

Specialty section:

This article was submitted to Microbial Immunology,

a section of the journal

Frontiers in Immunology

Received: 28 January 2021 Accepted: 07 June 2021

Published: 21 June 2021

Citation:

Francisco S, Arranz A, Merino J, Punzón C, Perona $R$ and Fresno $M$ (2021) Early p38 Activation Regulated

by MKP-1 Is Determinant for High

Levels of IL-10 Expression Through

TLR2 Activation.

Front. Immunol. 12:660065. doi: 10.3389/fimmu.2021.660065

\section{Early p38 Activation Regulated by MKP-1 Is Determinant for High Levels of IL-10 Expression Through TLR2 Activation}

\author{
Sara Francisco ${ }^{1,2,3}$, Alicia Arranz ${ }^{3 \dagger}$, Javier Merino ${ }^{1,2,3}$, Carmen Punzón ${ }^{1,2}$, \\ Rosario Perona ${ }^{4}$ and Manuel Fresno ${ }^{1,2,3 *}$
}

${ }^{1}$ DIOMUNE S. L., Parque Científico de Madrid, Madrid, Spain, ${ }^{2}$ Centro de Biología Molecular Severo Ochoa, Universidad Autónoma de Madrid, Madrid, Spain, ${ }^{3}$ Centro de Biología Molecular "Severo Ochoa", Consejo Superior de Investigaciones Cientificas (CSIC)-Universidad Autónoma de Madrid, Madrid, Spain, ${ }^{4}$ Instituto de Investigaciones Biomedicas, Alberto Sols Universidad Autónoma de Madrid, Madrid, Spain

Toll-like receptors (TLRS) play a crucial role in the recognition of pathogen-derived components as a first line of defense against infections. It has been suggested that depending on the nature of the pathogens, TLRs activation induce a distinct cytokine profile that may contribute to the polarization of the acquired immune response. Here, we investigated the early MAPK signaling activation via TLR4 and TLR2 receptors and its impact in differential cytokine profile by macrophages. We found that TLR2 ligands activated MAPKs p38 and ERK earlier compared to the TLR4 ligand LPS in macrophages. Higher IL-10/IL-12 and IL-10/TNF- $\alpha$ ratios were also observed at later time points in response to TLR2 ligands compared to LPS. The results also indicate an earlier activation of the phosphatase MKP-1 and that MKP-1 KO macrophages show a prolongation in p38 phosphorylation in response to TLR2 stimulation. Furthermore, p38 is critical for IL-10 expression in response to TLR2 ligands, which triggers the macrophage change to a $\mathrm{M} 2$ and regulatory phenotype in contrast to the M1 phenotype induced by TLR4 activation. Therefore, the early TLR2-mediated p38 induction contributes for the high IL-10 production, likely as a virulence strategy to suppress host Th1 response against certain types of pathogens.

Keywords: macrophages, innate immunity, toll-like receptors, MAPK signaling, cytokines

\section{INTRODUCTION}

Innate immune mechanisms that follow recognition of microbe or danger signals influence the nature and magnitude of subsequent adaptive immune responses (1). Toll-like receptors (TLRs) are key components of the immune system with unique capacity to sense the presence of pathogens through binding to a vast array of conserved microbial components from bacterial, viral and fungal origin named pathogen-associated molecular patterns (PAMPs). This recognition triggers the activation of intracellular signaling pathways determinant to mount a rapid and effective immune response $(2,3)$. 
Surface TLRs, especially TLR2 and TLR4 have gained importance due to their ability of recognize a diversified array of pathogenic ligands. TLR4 is known to complex with MD-2 and recognize the lipopolysaccharide LPS molecule, present in the gram-negative bacteria cell wall (4). TLR2 recognizes a wide array of ligands as lipopeptides, lipoteichoic acid from Grampositive bacteria, lipoarabinomannan from mycobacteria, zymosan from fungi, tGPI-mucin from Trypanosoma cruzi and the hemagglutinin protein from measles virus (4). This receptor forms an m-shaped heterodimer with either TLR1 or TLR6 or recognition of tri- or di acylated lipopeptides respectively $(5,6)$.

Upon binding of microbial products, TLRs trigger a cascade of signals that induce the production of several cytokines determinant for pathogen killing and activation of the adaptive immunity system. Several previous studies have demonstrated that signaling by distinct TLRs is not fully equivalent leading to differential cytokine gene induction (7). Indeed, TLR4 agonists induced mostly proinflammatory cytokines such as TNF, IL-12 and IFN- $\beta$ in contrast to TLR2 ligands, which induced preferentially IL-10, IL-8 and IL-23 in human DCs (8).

The activity of the MAPKs ERK and p38 are determinant in eliciting inflammatory responses through activation of multiple transcription factors and through the stabilization and translation of cytokines mRNA that contain AU-rich elements (9). MAP kinases are primarily inactivated by a group of dualspecificity protein phosphatases through dephosphorylation of the critical tyrosine and threonine residues of activated MAP kinases. Therefore, these phosphatases may serve as crucial feedback control regulators in the innate immune response during microbial infection. The phosphatase MKP-1 is highly expressed in macrophages and studies with MKP-1 KO peritoneal macrophages show that this phosphatase regulates MAPKs p38 and JNK with little effect on ERK $(10,11)$. Moreover, recent studies have demonstrated that p38 is determinant for IL-10 expression in response to LPS $(12,13)$.

Considering the previous studies that demonstrate a differential cytokine profile between TLR2 and TLR4 activation, we prompted to investigate whether this differential cytokine pattern can be ascribed to differences on MAPKs signaling pathways activation by TLR4 and TLR2 ligands. In our study, we demonstrate that the TLR2 ligands Pam3CSK4 (TLR2/1) and FSL-1 (TLR2/6) induce higher levels of antiinflammatory IL-10 and lower levels of pro-inflammatory cytokines IL-12 and TNF- $\alpha$ compared to TLR4 ligands in mouse macrophages. TLR2 ligands activate MAPKs p38 and ERK earlier compared to TLR4 ligands and MKP-1 controls p38 phosphorylation in response to TLR2 activation. These results suggest that early p38 activation is critical for the higher levels of IL-10 induction in response to TLR2 ligands.

\section{MATERIALS AND METHODS}

\section{Reagents}

TLR4 ligand LPS from E. coli O111:B4 (Sigma), TLR2/TLR1 ligand Pam3CSK4 and TLR2/6 ligand FSL-1 (InvivoGen) were resuspended in sterile PBS 1x. The inhibitors SB203580 (Cayman), 5Z-7-oxozeaenol (Calbiochem) (Merck) and FR180204 (Merck) were resuspended in DMSO and diluted in cell culture media achieving a final DMSO concentration of $0,1 \%(\mathrm{v} / \mathrm{v})$.

\section{Cell Lines}

The murine macrophage cell line RAW264.7 was cultured in RPMI 1640 medium (Gibco) (2 mM L-glutamine, antibiotics: 100 units/mL penicillin, $100 \mu \mathrm{g} / \mathrm{mL}$ streptomycin), supplemented with 5\% FBS (Merck). Cells were cultured in 12well plates at a density of $0,5 \times 10^{6}$ cells/well and serum deprived for $16 \mathrm{~h}$ prior ligand stimulation.

\section{Isolation of Mouse Peritoneal Macrophages}

C57BL/6 WT, TLR2 and TLR4 KO mice were obtained from S. Akira and MKP-1 KO mice from Bristol-Myers Squibb. All mice were bred and maintained in the animal facilities of the Centro de Biologia Molecular Severo Ochoa in Universidad Autonoma de Madrid. All animal procedures were performed in strict accordance with the European Commission legislation for the protection of animal used purposes (2010/63/EU). The protocol for the treatment of the animals was approved by the Comite de Ética de la Dirección General del Medio Ambiente de la Comunidad de Madrid, Spain (permits PROEX 128/15). Thioglycolate-elicited peritoneal macrophages (PM) were isolated from 6-8-week-old pathogen-free mice. Cells were cultured in RPMI 1640 (2 mM L-glutamine, antibiotics 100 units/mL penicillin, $100 \mu \mathrm{g} / \mathrm{mL}$ streptomycin) with 5\% FBS and seeded into 6-well-pates at a density of $1 \times 10^{6}$ cells/well. Cells were allowed to adhere for $2 \mathrm{~h}$ and then the medium was changed to remove non-adherent cells. After $24 \mathrm{~h}$, medium was replaced with new complete medium prior treatment.

\section{mRNA Isolation and RT-qPCR}

Total cellular RNA was isolated using NZyol Reagent (NZYTech). cDNA was prepared by reverse transcription (GoTaq 2-Step RT-qPCR System, Promega) and amplified by PCR using SYBR ${ }^{\circledR}$ Green PCR Master Mix and ABI Prism 7900HT sequence detection system (Applied Biosystems), with the following primers: mTNF- $\alpha$ F: CCACCACGCTCTTCTGTC TAC; R: AGGGTCTGGGCCATAGAACT; mIL-10 F: ATCGAT TTCTCCCCTGTGAA; R: TGTCAAATTCATTCATGGCCT; mIL-12p40 F: TGGTTGCCATCGTTTTCCTG; R: ACAGGTGA GGTTCACTGTTTCT; mMKP-1 F: CTACCAGTACAAGAGC ATCCC; R: AACTCAAAGGCCTCGTCCAG; mRPL13A F: ATCCCTCCACCCTATGACAA; R: GCCCCAGGTAAGCAAA CTT. The $2-\Delta \Delta \mathrm{Ct}$ method was applied to analyze the relative changes in expression profiling and all quantifications were normalized to the housekeeping gene RPL13A.

\section{ELISA}

Cytokine concentration was determined for IL-10, TNF- $\alpha$, IL2p40 using DuoSet ELISA kit from R\&D systems, according to the manufacturers protocol. 


\section{Western Blot}

Cells were lysed in ice-cold lysis buffer (50 mM Tris pH 7,5, 150 $\mathrm{mM} \mathrm{NaCl}, 1 \%$ Triton X-100, $1 \mathrm{mM}$ EDTA, 10\% Glycerol), Phosphatase and Protease inhibitors (78440 Thermo Fisher). Equal protein amount $(20 \mu \mathrm{g})$ from each cell lysate was separated on SDS $10 \%$ polyacrylamide gel and transferred to a nitrocellulose membrane (Bio-Rad). Membranes were blocked with 3\% BSA for $1 \mathrm{~h}$ and incubated with the with antibodies against p-p38 (Thr180/Tyr182) (9211) (1:1000), total p38 (9212) (1:1000), p-ERK1/2 (Thr202/Tyr204) (9101) (1:1000), total ERK 1/2 (9102) (1:1000) from Cell Signaling, MKP-1 V-15 (sc-1199) (1:500) and $\beta$-actin (sc-47778) (1:2000) from Santa Cruz Biotechnology. The membranes were then incubated with the respective HPR-conjugated secondary antibody for $1 \mathrm{~h}$ and developed using ECL substrate (BioRad).

\section{Statistics}

Analysis was performed using GraphPad Prism 5 software. Quantitative results are expressed as means \pm SEM or mean \pm $\mathrm{SD}$. Statistical analysis was performed using one-way ANOVA followed by Bonferroni multiple comparisons. A p-value less than 0.05 was considered statistically significant

\section{RESULTS}

\section{TLR2 Ligands Induce Higher Levels of IL-10 than TLR4 Ligand LPS}

TLR's activation by various microbial-derived components induce distinct cytokine responses in macrophages that may fine-tune the acquired immune response. To determine whether TLR2 and TLR4 differ in their ability to activate macrophage responses, Raw264.7 cells were stimulated with specific ligands for TLR4 (LPS), TLR2/1 (Pam3CSK4) and TLR2/6 (FSL-1). The LPS stimulus induced higher transcription levels of IL-12p40 and TNF- $\alpha$ but lower induction of IL-10 cytokine than TLR2 ligands (Figure 1). This was observed over a wide range of ligand concentrations (Supplementary Figure 1). On the other hand, TLR2 ligands induced higher levels of IL-10, but lower activation of proinflammatory cytokines than LPS. Differences in IL-10 and IL12 levels are more evident after 24 hours of stimulation which is the peak time for their secretion, as the opposite of TNF- $\alpha$ which is an earlier secreted cytokine (Supplementary Figure 2). To ensure that the differential cytokine induction observed in Raw264.7 cells was not a cell-line occurrence, the same experiment was performed using wild-type peritoneal macrophages (Figure 2). When these cells were treated with LPS, Pam3CSK4 or FSL-1, a similar pattern on cytokine's induction was observed by TLR4 and TLR2 activation. This indicates that TLR2 ligands induce a more anti-inflammatory response compared to TLR4 ligands observed by the higher IL$10 / I L-12$ p 40 and IL-10/TNF- $\alpha$ ratios. These results demonstrate that both macrophage cell types (cell line vs primary cell) have a similar cytokine induction profile via TLR4 and TLR2 receptors.

\section{TLR2 Activation Induces p38 and ERK Phosphorylation Earlier and Faster Compared to TLR4}

Since TLR4 and TLR2 receptor's activation induce a distinct cytokine pattern, the next step was to examine whether TLR4 and TLR2 ligands could differentially activate some of the cell signaling pathways. Raw264.7 cells were serum starved and then stimulated for 3, 10, 30 and 60 minutes with LPS, Pam3CSK4 or FSL-1. We detected MAPKs ERK and p38 activation in response to LPS, observing phosphorylation around 30 and $60 \mathrm{~min}$ (Figure 2). Interestingly, TLR2 ligands activate MAPK signaling pathways faster; by 3 min after stimulation, phosphoERK and phospho-p38 bands were clearly visible. Furthermore, p38 and ERK phosphorylation induced by TLR2 declines faster, at 30 and 60 min compared with the TLR4 ligand LPS, which still maintains at $60 \mathrm{~min}$. For longer time points p38 shows more activation phases (phosphorylation band at 2,15, and $24 \mathrm{~h}$ ) similar for LPS, Pam3CSK4 and FSL-1 stimulation (Supplementary Figure 3). This indicates a differential p38 activation by TLR4 and TLR2 ligands in the first $60 \mathrm{~min}$ of stimulation. In peritoneal macrophages, a similar early kinetic signature was observed (Supplementary Figure 4). Peritoneal macrophages from TLR2 KO and TLR4 KO mice were also used to confirm the ligands stimulation-specificity. Upon stimulation of TLR2 KO macrophages, TLR2 ligands were unable to activate MAPK pathways, indicating the specificity of these ligands for TLR2 receptor. As expected, LPS was able to activate these cells but not TLR4 KO macrophages

To study the differential kinetic signaling between TLR4 and TLR2 ligands, special focus was given to investigate whether different upstream ERK and p38 activators could be induced via TLR4 or TLR2 activation. To this end, macrophages were pretreated with TAK1 inhibitor (5Z-7-oxzeaenol) and MEK 1/ 2 inhibitor (U0126) once these mediators are described to be implicated in ERK activation. In the presence of TAK1 inhibitor, ERK and p38 phosphorylation were strongly reduced upon stimulation with TLR4 and TLR2 ligands, both in peritoneal macrophages (Figure 3A) and Raw264.7 cells (Supplementary Figure 5A) suggesting that TAK1 is an upstream activator for these MAPKs. Similarly, the inhibition of MEK by U0126 produced similar results with all ligands in Peritoneal (Figure 3B) and Raw264.7 cells (Supplementary Figure 5B)

\section{MKP-1 Is Activated Earlier by TLR2 Activation and Controls TLR2-Induced p38 Phosphorylation}

Since no clear effect in upstream kinase(s), and to further understand the cause of differential kinetics on p38 and ERK rapid phosphorylation by TLR2 activation, we addressed the possible role of phosphatases, more specifically MKP-1. This phosphatase was described to be induced and stabilized through phosphorylation by ERK in LPS-stimulated cells. Moreover, MKP-1 has higher affinity to dephosphorylate p38 and JNK rather than ERK (14). Stimulation of Raw264.7 cells and peritoneal macrophages with the TLR ligands exhibit MKP-1 induction (Figure 4A). In response to LPS, MKP-1 was induced 

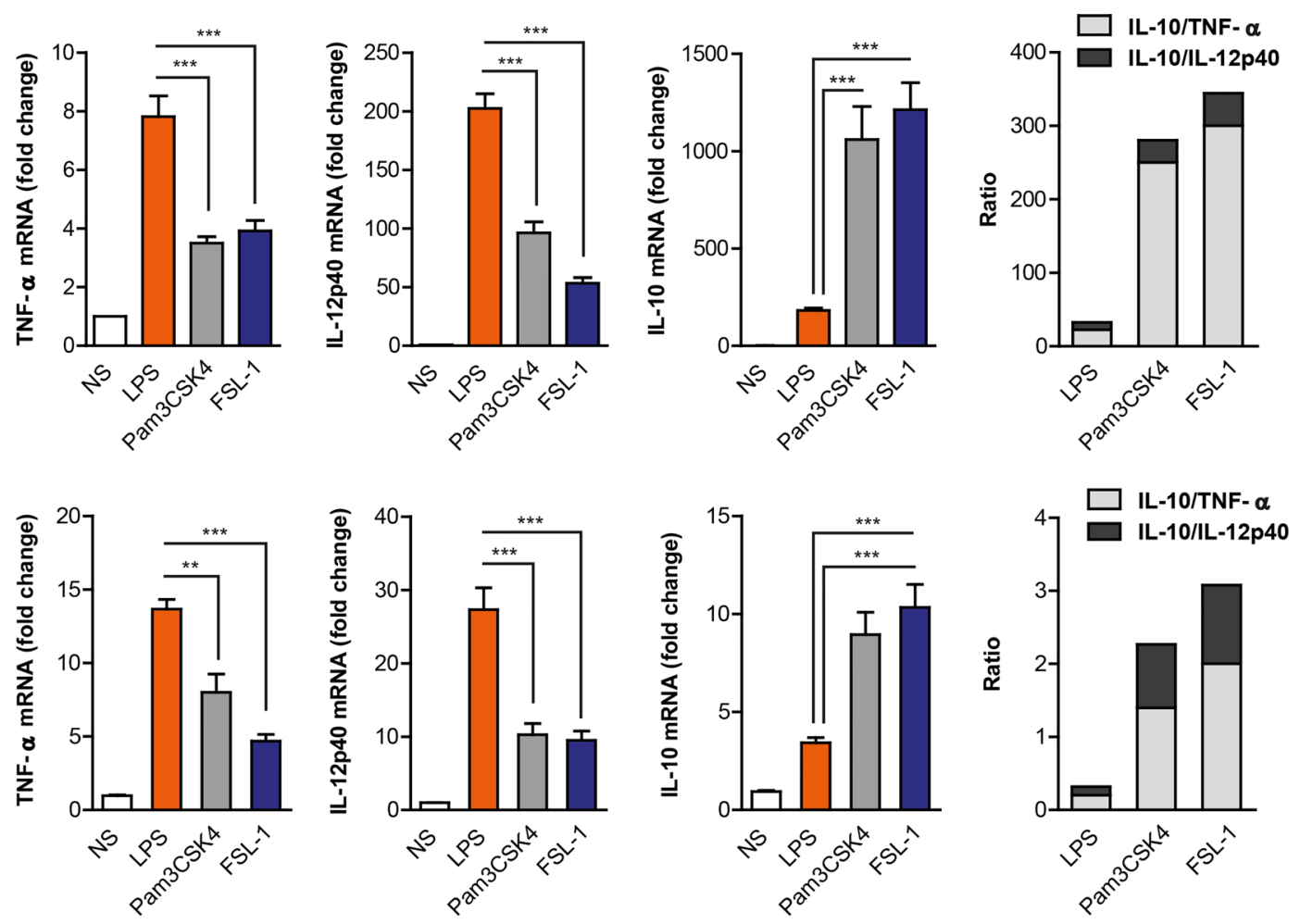

FIGURE 1 | TLR4 and TLR2 ligands induce a distinct cytokine expression pattern in Raw264.7 cell line and peritoneal macrophages. Raw264.7 cells (upper graph) and mouse peritoneal macrophages (lower graph) were stimulated with LPS (100 ng/mL), Pam3CSK4 (1 $\mu \mathrm{g} / \mathrm{mL})$ and FSL-1 (100 ng/mL) for 24 h. Cytokine's mRNA expression was assayed by quantitative RT-PCR, normalized to RPL13A, and presented relative to unstimulated cells (NS). Data shown is from a representative experiment of the three independent experiments performed from triplicate cultures (mean $\pm \mathrm{SEM})\left({ }^{\star \star} \mathrm{p}<0.01\right.$ and $\left.{ }^{\star \star \star} \mathrm{p}<0.001\right)$.

at $60 \mathrm{~min}$ after stimulation, whereas in response to TLR2 ligands MKP-1 was detected earlier, at $30 \mathrm{~min}$. A similar kinetic pattern was observed for MKP-1 transcription at 30 and $60 \mathrm{~min}$ (Figure 4B), however in peritoneal macrophages MKP-1 mRNA levels started to decay at 60 min (Supplementary Figure 6). This data indicates that TLR2 ligands induce MKP1 transcription and expression earlier compared to the TLR4 ligand LPS.

Previous data demonstrated that MKP-1 controls p38 activation in response to TLR4 activation $(13,15)$. Therefore, the MKP-1 role in MAPKs activation in response to FSL-1 was addressed. WT and MKP1-deficient macrophages were treated with FSL-1 for $60 \mathrm{~min}$. Macrophages deficient in MKP-1 exhibited a prolongation of $\mathrm{p} 38$ phosphorylation but not for ERK comparing with WT (Figure 4C). Therefore, besides TLR4, MKP-1 will preferably regulate $\mathrm{p} 38$ phosphorylation in response to TLR2/6 stimulation.

\section{Early p38 Activation Is Determinant for IL-10 Expression by TLR2 Stimulation}

Published data describes that IL-10 expression is dependent of p38 activation in response to LPS $(12,13,16)$. Thus, the next step was to investigate the effect of p38 and ERK inhibition in the cytokine's induction by the TLR2 ligands. A time-course kinetics was performed to determine the changes of cytokine levels in
Raw264.7 cells pretreated with p38 and ERK inhibitors, SB203580 or FR180204 respectively, and stimulated with TLR4 or TLR2 ligands for 4, 8 and 24 hours (Figure 5A). Stimulation with all TLR ligands gave a similar pattern for TNF- $\alpha$ induction. However IL- 6 levels by TLR2 ligands were lower at earlier time points compared to the levels induced by LPS At 24 hours, p38 inhibition resulted in TNF- $\alpha$ and IL- 6 induction in response to Pam3CSK4 and FSL-1, similar to the effect observed in response to LPS. More importantly, IL-10 levels are highly increased at 24 hours in response to TLR2 ligands compared to TLR4, being this effect fully dependent of p38. Inhibition of ERK produced variable effect depending on the time point tested. To confirm that early p38 activation by TLR2 ligands is decisive for the high levels of IL-10 expression, Raw264.7 cells were treated with p38 inhibitor $60 \mathrm{~min}$ after TLR stimulation with LPS, Pam3CSK4 or FSL-1 (Figure 5B). The inhibitor addition $1 \mathrm{~h}$ post TLR stimulation does not impair IL-10 levels, indicating that the early p38 activation is determinant for the high IL-10 expression levels observed for TLR2 stimulation.

The effect of p38 and ERK inhibition on cytokine's expression was further evaluated in peritoneal macrophages pretreated with p38 and ERK inhibitors and stimulated with TLR ligands for 24 hours. Interestingly, IL-12 was not affected in the presence of the p38 inhibitor in response to all TLR ligands and proinflammatory TNF- $\alpha$ only weakly in response to LPS 

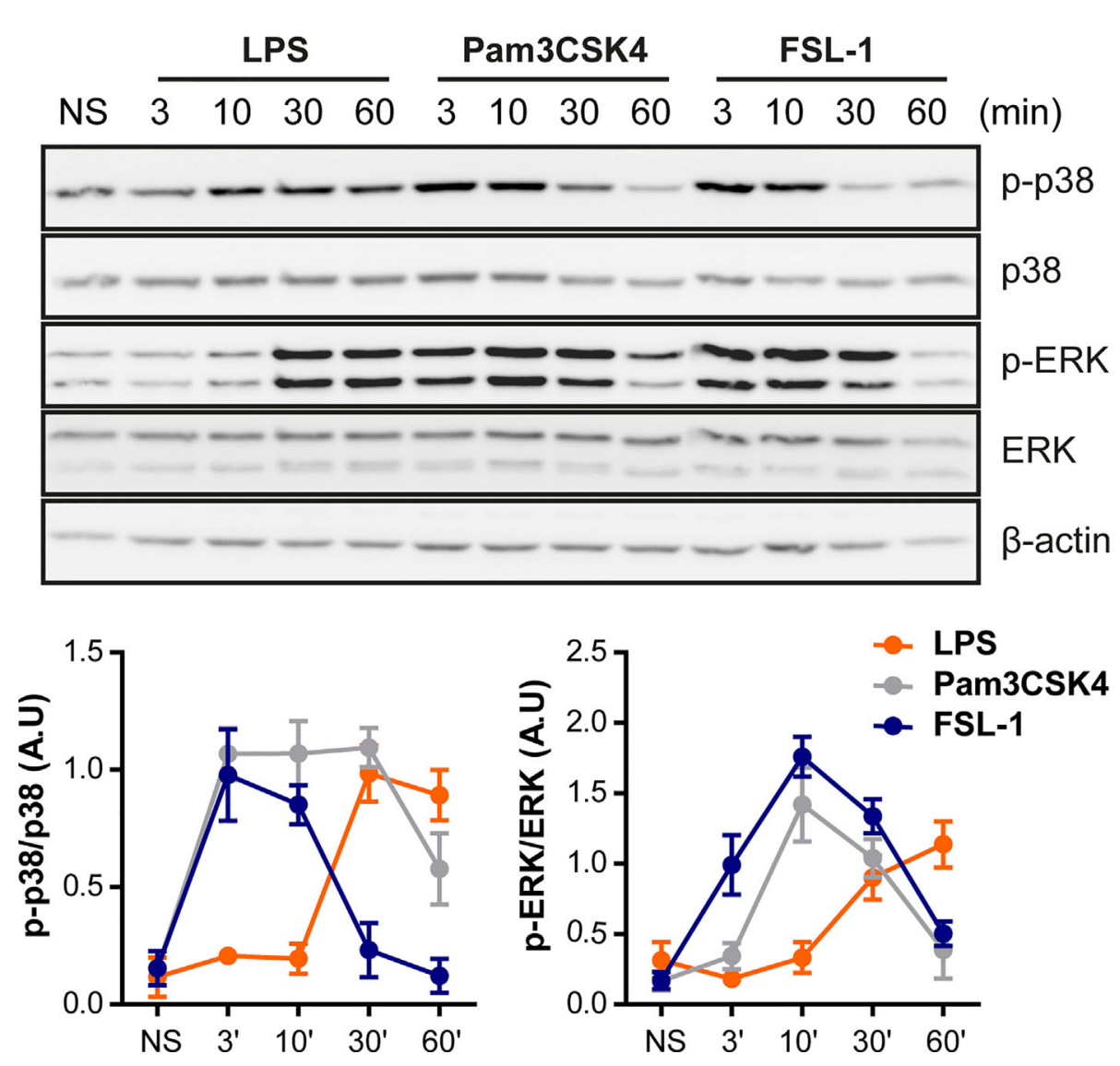

FIGURE 2 | TLR4 and TLR2 ligands exhibit different kinetics on MAPK pathways activation. Raw264.7 cells were treated with LPS (100 ng/mL), Pam3CSK4 (1 $\mu \mathrm{g} /$ $\mathrm{mL}$ ) and FSL-1 (100 ng/mL) at 3, 10, 30 and 60 min and stained with the antibodies indicated on the right side of the panels. The graphics below show the quantification of band intensities of the Western blots. The results are shown as mean \pm SEM of three independent experiments.

A

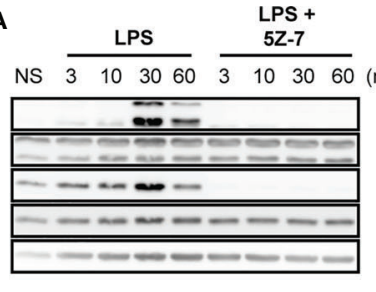

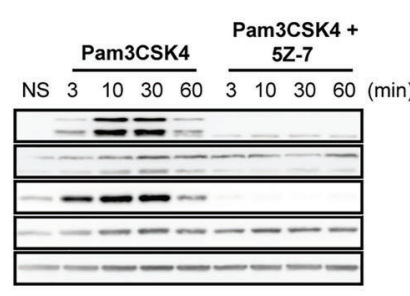

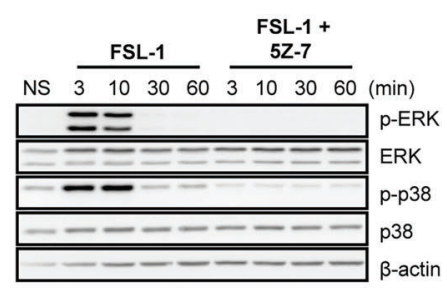

B

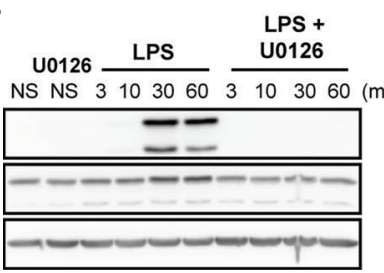

Pam3CSK4+ U0126 Pam3CSK4 U0126 NS NS $3 \longdiv { 1 0 3 0 6 0 } \overline { 3 1 0 3 0 6 0 } ( \mathrm { min } )$

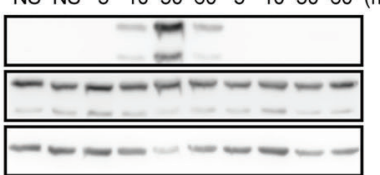

FSL-1 +

U0126 FSL-1 U0126

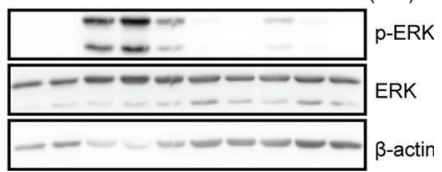

NS NS $3 \longdiv { 1 0 3 0 6 0 } \overline { 3 1 0 3 0 6 0 } ( \mathrm { min } )$

FIGURE 3 | TAK1 and MEK1/2 are upstream activators of ERK and p38 regardless of the TLR stimuli in peritoneal macrophages. Peritoneal macrophages were pretreated with $1 \mu \mathrm{M}$ 5Z-7-oxzeaenol ("5Z-7") (A) or with $1 \mu \mathrm{M} \cup 0126$ (B) for 30 min. Cells were left unstimulated (NS) or stimulated with LPS (100 ng/mL), Pam3CSK4 $(1 \mathrm{\mu g} / \mathrm{mL})$ and FSL-1 $(100 \mathrm{ng} / \mathrm{mL})$ at 3, 10, 30 and $60 \mathrm{~min}$. A representative western blot is shown of three independent experiments. 

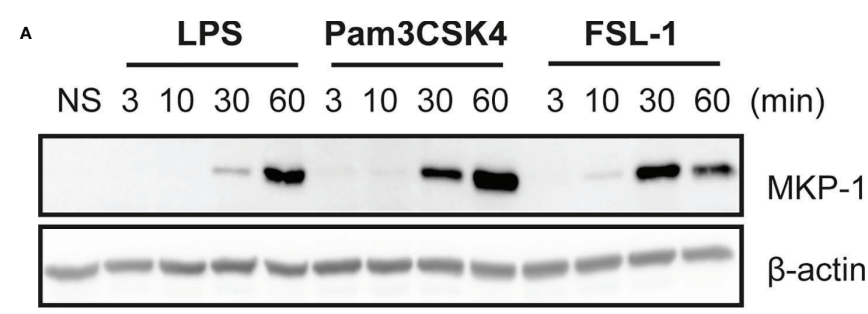

B
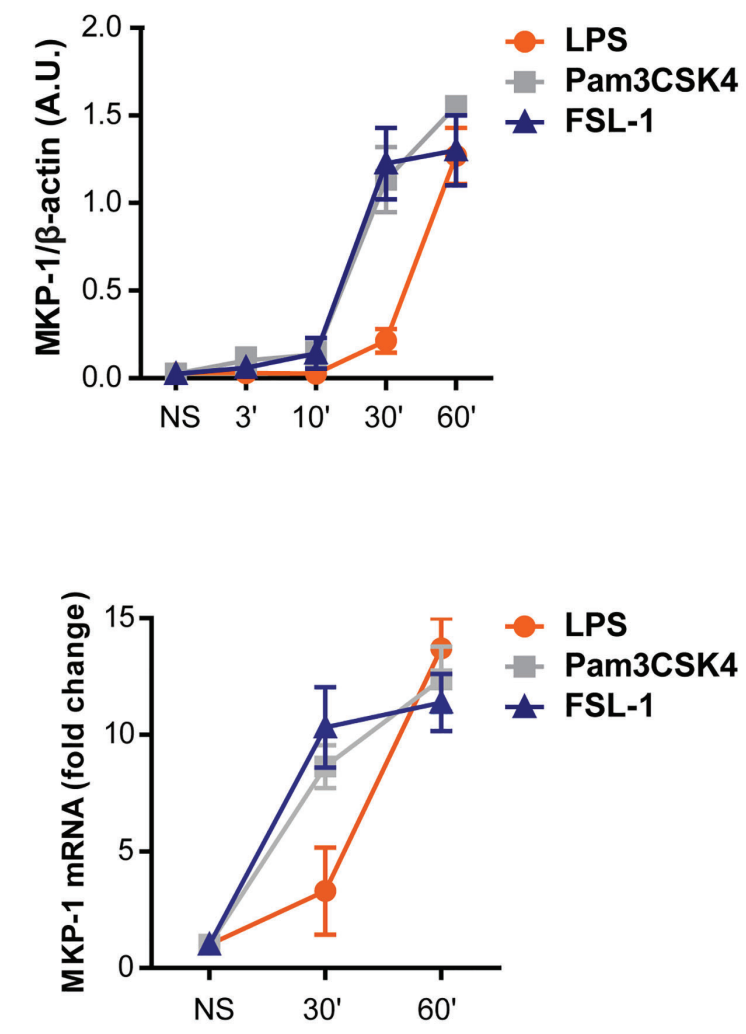

c

WT

MKP-1 KO

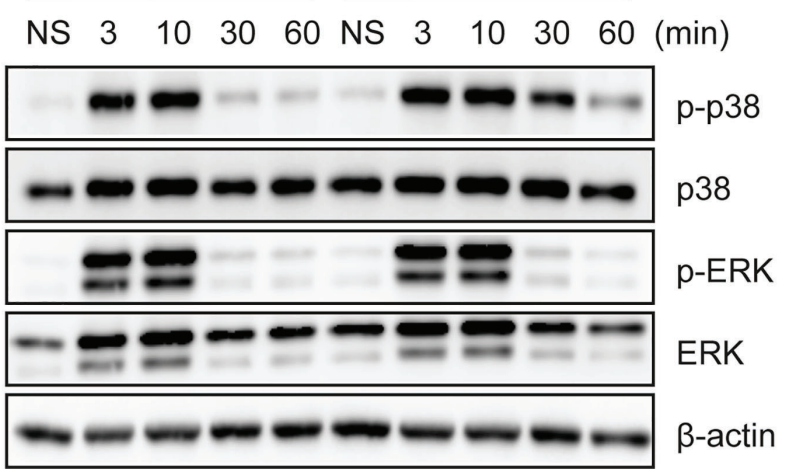

FIGURE 4 | MKP-1 is activated earlier by TLR2 activation and controls TLR2-induced p38 phosphorylation. (A) Raw264.7 cells were left unstimulated (NS) or stimulated with LPS (100 ng/mL), Pam3CSK4 (1 $\mathrm{mg} / \mathrm{mL})$ and FSL-1 (100 ng/mL) at 3, 10, 30 and 60 min and protein expression was measured by Western blot. (B) MKP-1 mRNA levels were measured by qPCR after Raw 264.7 cells stimulation with the three ligands at 30 and 60 min. (C) WT and MKP-1 KO peritoneal macrophages were left unstimulated (NS) or stimulated FSL-1 (100 ng/mL) at 3, 10, 30 and 60 min. $\beta$-actin was used as protein loading control. Images are representative of two experiments. 
A
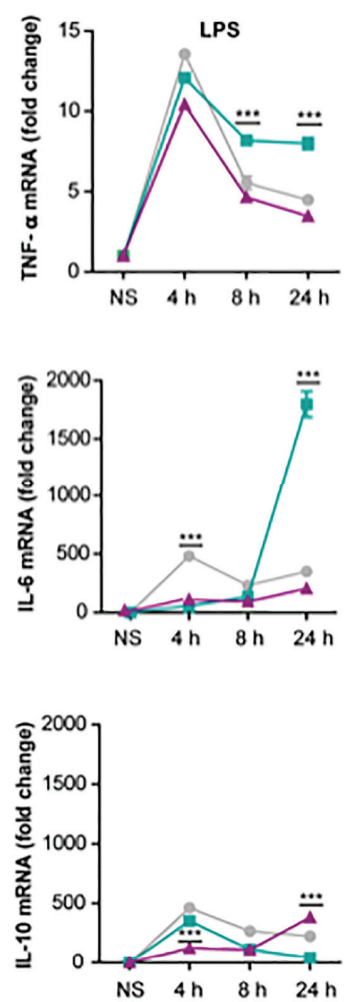
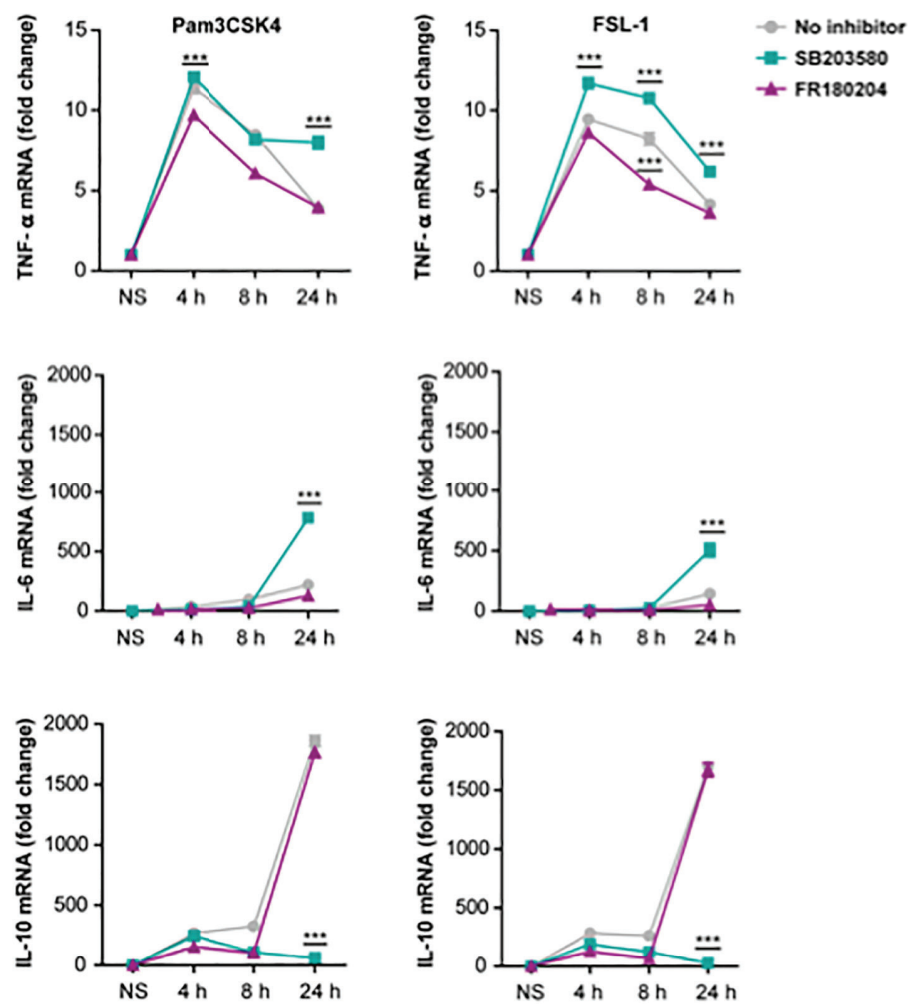
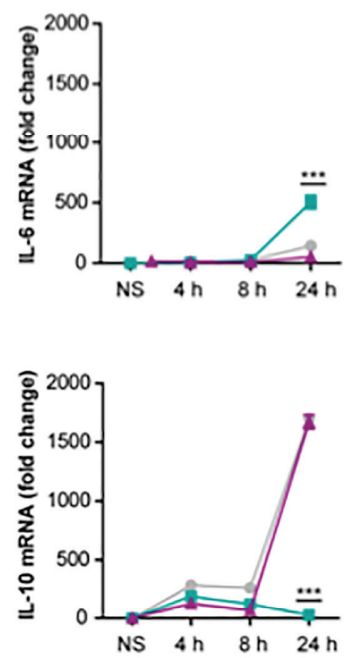

B

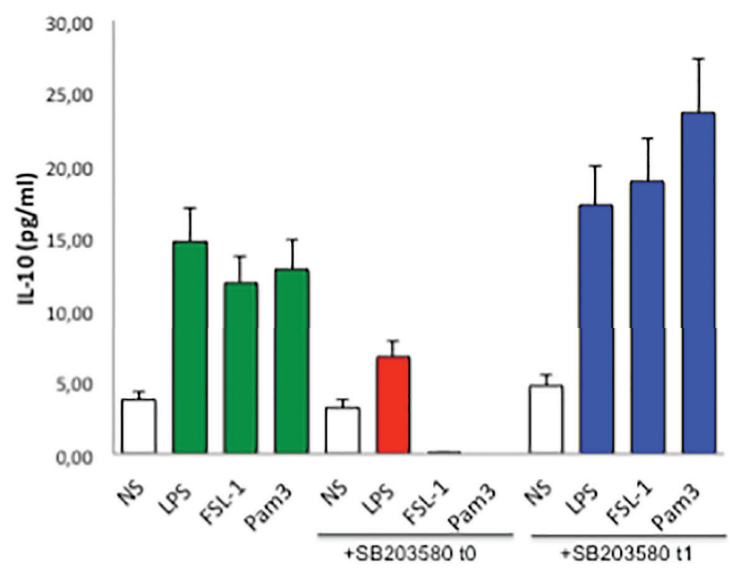

FIGURE 5 | Early p38 activation contributes for later IL-10 production in response to TLR2 ligands. (A) Raw264.7 cells were pre-treated with $10 \mu \mathrm{M}$ SB203580 or $10 \mu \mathrm{M}$ of FR180204 for $30 \mathrm{~min}$ and then stimulated with LPS (100 ng/mL), Pam3CSK4 (1 $\mathrm{mg} / \mathrm{mL})$ and FSL-1 (100 ng/mL) for 24 hours. Cytokines mRNA expression was assayed by quantitative RT-PCR, normalized to RPL13A, and presented relative to unstimulated cells (NS). Data from triplicate cultures are representative of three independent experiments (mean \pm SEM.) (** $\left.{ }^{\star \star}<0.001\right)$. (B) Raw264.7 cells were treated with $10 \mu \mathrm{M} \mathrm{SB203580}$ at the same time (t0) or 60 min (t1), after stimulation with LPS (100 ng/mL), Pam3CSK4 $(1 \mu \mathrm{g} / \mathrm{mL})$ and FSL-1 (100 ng/mL). Cell supernatants were collected after 24 hour-stimulation and IL-10 cytokine protein levels were measured by ELISA. Data are the mean of 2 experiments.

(Figure 6A). On the other hand, IL-10 levels were strongly decreased by p38 inhibition. A similar pattern was observed for the cytokine transcription levels in stimulated-peritoneal macrophages (Figure 6B). ERK inhibition by FR180204 had no effect on LPS-induced IL-10 although decreased slightly the production of this cytokine in response to TLR2 ligands (Figure 6A). Nonetheless, ERK inhibition affected TNF- $\alpha$ and IL12 production by LPS. These results imply that ERK and p38 are important for pro-inflammatory cytokines induction at early time points of inflammation, whereas p38 but not ERK contributes for 
later anti-inflammatory IL-10 production in response to TLR4 and TLR2 ligands.

\section{DISCUSSION}

Our results show that stimulation of macrophages with TLR4 or TLR2 ligands resulted in striking differences in cytokine production. Specifically, TLR2/6 and TLR2/1 ligands exhibit a higher induction of the anti-inflammatory mediator IL-10, whereas TLR4 favors the expression of pro-inflammatory or Th1 cytokines such as TNF- $\alpha$ and IL-12p40. The higher IL-10/ TNF- $\alpha$ and IL-10/IL-12p40 ratios observed in macrophages activated by TLR2 ligands suggest a change to a M2 and regulatory phenotype, whereas LPS induces an M1-type macrophage as previously described. This is concordant with previous published data in human DCs stimulated with LPS, PGN, zymosan and Pam3Cys, where TLR2 ligands favored a Th2 response in contrast to LPS (17-19). In this regard, several pathogens, such as Brucella abortus, Candida albicans or Mycobacterium tuberculosis may explore TLR2-mediated IL-10 induction as a virulence mechanism to induce M2 macrophages and suppress Th1 response against the pathogen (20,21). Given this distinct cytokine profile, it was questionable if TLR4 and TLR2 ligands could activate exactly similar signaling pathways, including the activation MAPKs p38 and ERK. Our results demonstrate different kinetics of activation between TLR4 and TLR2 ligands, however Pam3CSK4 and FSL-1 show similar kinetics of activation, which is in accordance with previous studies (22). Our experiments were performed in both a macrophage cell line and primary macrophages, thus showing that this differential kinetics is an important property of TLR signaling in macrophages.

A possible explanation for the differential MAPKs activation could be that TLR4 and TLR2 ligands would activate different upstream mediators of p38 and ERK. TAK1 is a general upstream kinase described to be important for activation of NF- $\mathrm{BB}, \mathrm{p} 38$, JNK and ERK (23) Our results show that TLR4 and TLR2 ligands are dependent of TAK1 to induce ERK and p38 and this is observed for the Raw264.7 cell line as well as for peritoneal macrophages. Moreover, our results with U0126-specific MEK1/2 is inhibitor also suggested that no major differences in the activation of this pathway by TLR2 an TLR ligands may be responsible for differential ERK activation. Many pathways could be potential mediators of this differential early signaling between TLR2 and TLR4 ligands, including TPL2, Ras, PKC zeta, PI3K, etc., that deserves further research.

On the other hand, dephosphorylation of MAPKs is one of the most efficient mechanisms to control their activation. Dual specificity phosphatases (DUSP), also termed MKP can negatively regulate MAPKs activation through dephosphorylation of phosphotyrosine and phosphothreonine residues (24). DUSPs can be induced by TLR-mediated activation of MAPKs, creating a negative feedback loop to limit MAPKs activation. Among DUSPs, MKP-1 is highly expressed in macrophages (25). Our results show that both TLR4 and TLR2 ligands induce MKP-1, however with different kinetics of activation, similarly to ERK and p38 MAPKs. TLR2/1 and TLR2/6 induced a faster activation of the
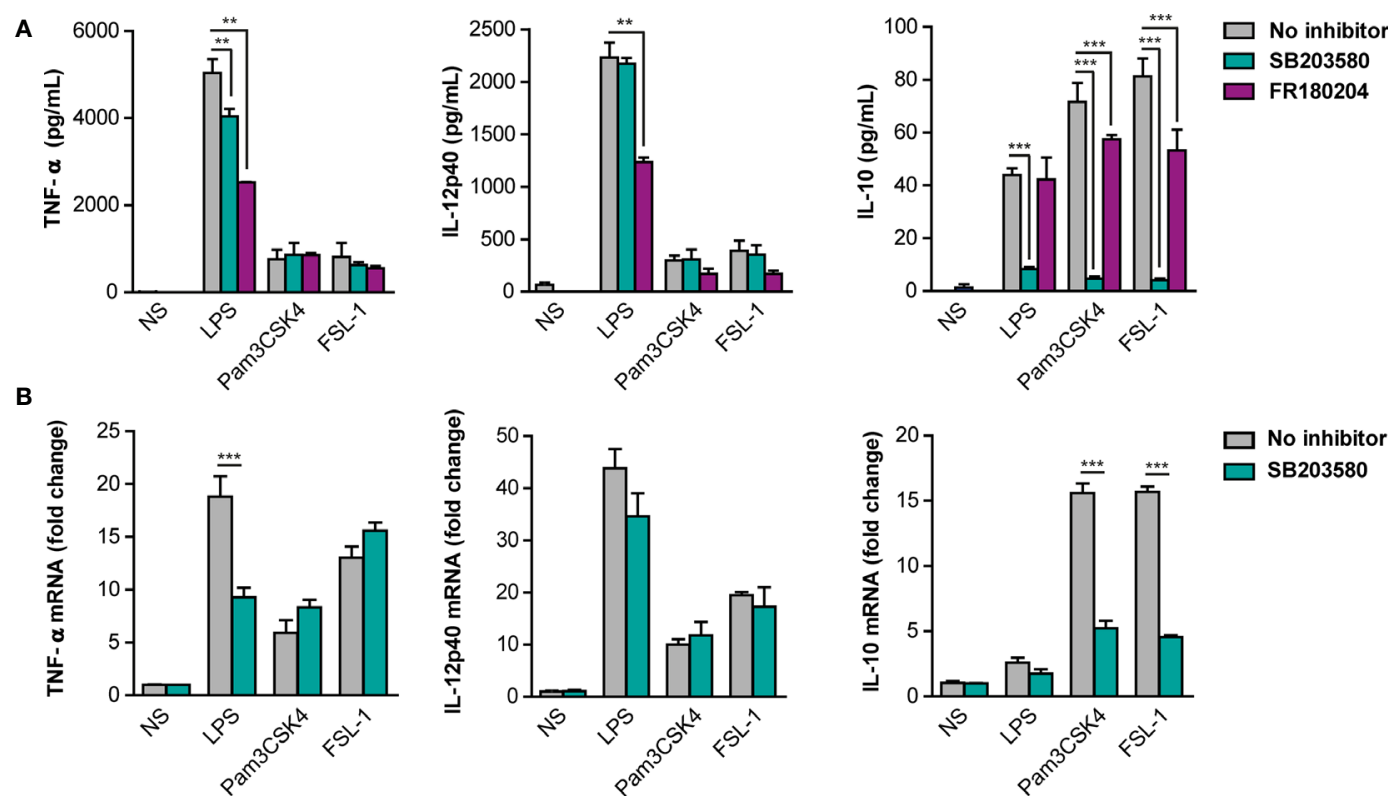

FIGURE 6 | Differential contribution of p38 and ERK for cytokine production in response to TLR ligands. Peritoneal macrophages were pre-treated with $10 \mu \mathrm{M}$ SB203580 or 10 MM FR180204 for 30 min and then stimulated with LPS (100 ng/mL), Pam3CSK4 (1 $\mu \mathrm{g} / \mathrm{mL})$ and FSL-1 (100 ng/mL) for 24 hours. (A) Cytokine protein levels and (B) Cytokine mRNA levels were measured by ELISA and qPCR respectively. Data from triplicate cultures (mean \pm SEM.) are representative of one of the three independent experiments performed $\left({ }^{\star \star} p<0.01\right.$ and $\left.{ }^{\star \star \star} p<0.001\right)$. 
MKP1 phosphatase than TLR4 coincident with the sudden drop in phosphorylated ERK. Thus, it is likely that this temporal difference in MKP-1 induction via TLR4 and TLR2 may be responsible for the same differences observed in ERK and p38 phosphorylation.

MKP-1 induction is determinant for controlling the length of MAPK responses in macrophages. Previous studies demonstrated that MKP-1 KO peritoneal, alveolar macrophages and bone marrow-derived macrophages showed prolonged activation of p38 and JNK, suggesting that MKP-1 regulates primarily p38 and JNK, but has little effect on ERK, in response to LPS $(13,26,27)$. Our results show a similar result in response to the TLR2 ligand FSL-1, where MKP-1 KO PM exhibit a prolonged p38 phosphorylation whereas ERK phosphorylation was not affected. In the absence of MKP-1, p38 is likely to eventually become inactivated by other MKPs, though at a much slower rate. This is consistent with the observation that deactivation of $\mathrm{p} 38$ in MKP-1 KO macrophages was delayed. Overall, MKP-1 has a central role in the regulation of $\mathrm{p} 38$ by TLR2 signaling in macrophages.

Importantly, our results also demonstrate that p38 is critical for later anti-inflammatory IL-10 induction but not for proinflammatory or Th1 cytokines, regardless of the stimuli. This is in accordance with Chi et al, which demonstrates that IL-10 induction mediated by LPS was blocked by a p38 inhibitor, implying that p38 mediates IL-10 synthesis (13). Our results indicate that TLR2 ligands also induce IL-10 expression fully dependent of p38 at later stages. Importantly, the higher levels of IL-10 expression induced by TLR2 ligands were proved to be dependent on early activation of $\mathrm{p} 38$, in contrast with the later activation by LPS. The kinetics of $\mathrm{p} 38$ blockade indicates that this window period $0-1 \mathrm{~h}$ is crucial for sequential IL10 production. Furthermore, it is described that IL-10 inhibits the transcription of pro-inflammatory cytokines via IL10R-Janus kinase-STAT3 pathway (28). Therefore, the earlier activation of this pathway could contribute for the higher IL-10 levels and lower proinflammatory cytokines observed via TLR2 activation. Transcription factors also influence cytokine expression. For example, SP1, c/EBP $\beta, \mathrm{CREB} / \mathrm{AP} 1$ and MAF have been shown to bind to IL-10 promoter. MSK1/MSK2-mediated activation of CREB and ATF1 induced IL-10 in response to LPS (29). However, the different stimuli may induce different transcription factors activated in a same cell type. For example, p38 induce IL-10 promoter via SP1 in response to LPS, whereas c/EBP5 was found to be recruited in response to cAMP $(12,30)$. Further studies are required to identify the downstream targets activated by early p38 phosphorylation that mediate IL-10 regulation in response to TLR2 ligands.

In addition, ERK activation seems to mediate some of the responses of TLR4, mostly pro-inflammatory or Th1 cytokines, since ERK inhibitors prevent partially LPS response. However, ERK activation did not affect on TLR2/1 and TLR2/6 mediated proinflammatory or Th1 cytokines and slightly IL-10 cytokine induction. This point out to a different involvement of MAPK, ERK and p38 in cytokine regulation depending on the nature of the foreign invader pathogen sensed by different TLRs.

In summary, our results indicate that TLR2 ligands induce higher levels of anti-inflammatory IL-10 and lower levels of proinflammatory cytokines IL-12, TNF- $\alpha$ compared to TLR4 ligands in macrophages. Our results support a model (Figure 7) in which TLR2 activates MAPKs faster and MKP-1 controls p38 phosphorylation which differentially controls pro and antiinflammatory cytokines. Our work shows for the first time that early and fast p38 activation in response to TLR2 ligands is critical

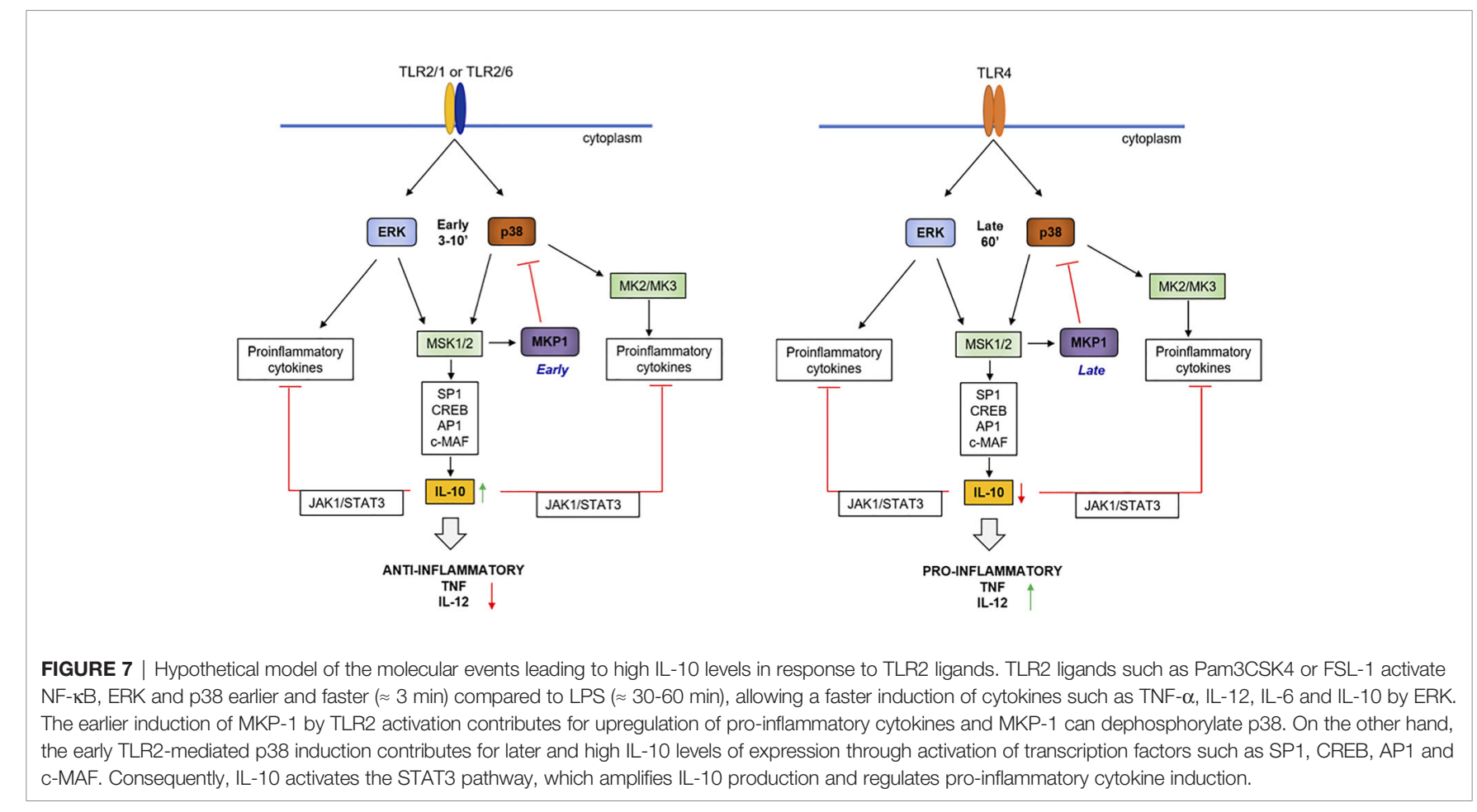


for the higher levels of IL-10 induction compared to TLR4, leading to reduced pro-inflammatory cytokines.

\section{DATA AVAILABILITY STATEMENT}

The original contributions presented in the study are included in the article/Supplementary Material. Further inquiries can be directed to the corresponding author.

\section{ETHICS STATEMENT}

The animal study was reviewed and approved by Comite de Ética de la Dirección General del Medio Ambiente de la Comunidad de Madrid, Spain (permits PROEX 128/15).

\section{AUTHOR CONTRIBUTIONS}

SF, AA, and MF designed the experiments. SF, CP, and AA performed the experiments. RP helped with macrophage $\mathrm{KO}$ mice experiments. SF and MF wrote the manuscript. All authors contributed to the article and approved the submitted version.

\section{REFERENCES}

1. Schenten D, Medzhitov R. The Control of Adaptive Immune Responses by The Innate Immune System. Adv Immunol (2011) 109:87-124. doi: 10.1016/ B978-0-12-387664-5.00003-0

2. Akira S, Takeda K. Toll-Like Receptor Signalling. Nat Rev Immunol (2004) 4 (7):499-511. doi: 10.1038/nri1391

3. Janeway CAJr, Medzhitov R. Innate Immune Recognition. Annu Rev Immunol (2002) 20:197-216. doi: 10.1146/annurev.immunol.20.083001.084359

4. Kawai T, Akira S. Toll-Like Receptors and Their Crosstalk With Other Innate Receptors in Infection and Immunity. Immunity (2011) 34(5):637-50. doi: 10.1016/j.immuni.2011.05.006

5. Jin MS, Kim SE, Heo JY, Lee ME, Kim HM, Paik SG, et al. Crystal Structure of the TLR1-TLR2 Heterodimer Induced by Binding of a Tri-Acylated Lipopeptide. Cell (2007) 130(6):1071-82. doi: 10.1016/j.cell.2007.09.008

6. Kang JY, Nan X, Jin MS, Youn SJ, Ryu YH, Mah S, et al. Recognition of Lipopeptide Patterns by Toll-like Receptor 2-Toll-like Receptor 6 Heterodimer. Immunity (2009) 31(6):873-84. doi: 10.1016/j.immuni. 2009.09.018

7. Hirschfeld M, Weis JJ, Toshchakov V, Salkowski CA, Cody MJ, Ward DC, et al. Signaling by Toll-Like Receptor 2 and 4 Agonists Results in Differential Gene Expression in Murine Macrophages. Infect Immun (2001) 69(3):147782. doi: 10.1128/IAI.69.3.1477-1482.2001

8. Re F, Strominger JL. Il-10 Released by Concomitant TLR2 Stimulation Blocks the Induction of a Subset of Th1 Cytokines That are Specifically Induced by TLR4 or TLR3 in Human Dendritic Cells. J Immunol (2004) 173(12):7548-55. doi: 10.4049/jimmunol.173.12.7548

9. Dong C, Davis RJ, Flavell RA. MAP Kinases in the Immune Response. Annu Rev Immunol (2002) 20:55-72. doi: 10.1146/annurev.immunol.20.091301.131133

10. Wang X, Liu Y. Regulation of Innate Immune Response by MAP Kinase Phosphatase-1. Cell Signal (2007) 19(7):1372-82. doi: 10.1016/ j.cellsig.2007.03.013

11. Liu Y, Shepherd EG, Nelin LD. MAPK Phosphatases-Regulating the Immune Response. Nat Rev Immunol (2007) 7(3):202-12. doi: 10.1038/nri2035

12. Ma W, Lim W, Gee K, Aucoin S, Nandan D, Kozlowski M, et al. The p38 Mitogen-Activated Kinase Pathway Regulates the Human Interleukin-10

\section{FUNDING}

This research was funded by grants from European Unión "Host-microbe interactions in Health and disease. Interface with the immune system". HOMIN - 317057 - FP7-PEOPLE2012-ITN to Diomune and Ministerio de Ciencia e Innovación (SAF2013-42850-R and SAF2016-75988-R), Instituto de salud Carlos III “ (BIOIMID and RD16/0027/0006), Comunidad de Madrid (S2017/BMD-3671. INFLAMUNE-CM) to MF, and Institutional grants from "Fundación Ramón Areces" and "Banco de Santander".

\section{ACKNOWLEDGMENTS}

We acknowledge Maria Chorro and Mari Carmen Maza for excellent technical support.

\section{SUPPLEMENTARY MATERIAL}

The Supplementary Material for this article can be found online at: https://www.frontiersin.org/articles/10.3389/fimmu.2021.660065/ full\#supplementary-material

Promoter Via the Activation of Spl Transcription Factor in Lipopolysaccharide-Stimulated Human Macrophages. J Biol Chem (2001) 276(17):13664-74. doi: 10.1074/jbc.M011157200

13. Chi H, Barry SP, Roth RJ, Wu JJ, Jones EA, Bennett AM, et al. Dynamic Regulation of Pro- and Anti-Inflammatory Cytokines by MAPK Phosphatase 1 (MKP-1) in Innate Immune Responses. Proc Natl Acad Sci U S A (2006) 103 (7):2274-9. doi: 10.1073/pnas.0510965103

14. Brondello JM, Pouyssegur J, McKenzie FR. Reduced MAP Kinase Phosphatase-1 Degradation After p42/p44MAPK-Dependent Phosphorylation. Science (1999) 286(5449):2514-7. doi: 10.1126/science.286.5449.2514

15. Zhao Q, Wang X, Nelin LD, Yao Y, Matta R, Manson ME, et al. MAP Kinase Phosphatase 1 Controls Innate Immune Responses and Suppresses Endotoxic Shock. J Exp Med (2006) 203(1):131-40. doi: 10.1084/jem.20051794

16. Kim C, Sano Y, Todorova K, Carlson BA, Arpa L, Celada A, et al. The Kinase p38 Alpha Serves Cell Type-Specific Inflammatory Functions in Skin Injury and Coordinates Pro- and Anti-Inflammatory Gene Expression. Nat Immunol (2008) 9(9):1019-27. doi: 10.1038/ni.1640

17. Agrawal S, Agrawal A, Doughty B, Gerwitz A, Blenis J, Van Dyke T, et al. Cutting Edge: Different Toll-Like Receptor Agonists Instruct Dendritic Cells to Induce Distinct Th Responses Via Differential Modulation of Extracellular Signal-Regulated Kinase-Mitogen-Activated Protein Kinase and C-Fos. J Immunol (2003) 171(10):4984-9. doi: 10.4049/jimmunol.171.10.4984

18. Dillon S, Agrawal S, Banerjee K, Letterio J, Denning TL, Oswald-Richter K, et al. Yeast Zymosan, a Stimulus for TLR2 and dectin-1, Induces Regulatory Antigen-Presenting Cells and Immunological Tolerance. J Clin Invest (2006) 116(4):916-28. doi: 10.1172/JCI27203

19. Re F, Strominger JL. Toll-Like Receptor 2 (TLR2) and TLR4 Differentially Activate Human Dendritic Cells. J Biol Chem (2001) 276(40):37692-9. doi: 10.1074/jbc.M105927200

20. Netea MG, Sutmuller R, Hermann C, van der Graaf CA, van der Meer JW, van Krieken JH, et al. Toll-Like Receptor 2 Suppresses Immunity Against Candida Albicans Through Induction of IL-10 and Regulatory T Cells. J Immuno (2004) 172(6):3712-8. doi: 10.4049/jimmunol.172.6.3712

21. Richardson ET, Shukla S, Sweet DR, Wearsch PA, Tsichlis PN, Boom WH, et al. Toll-Like Receptor 2-Dependent Extracellular Signal-Regulated Kinase Signaling in Mycobacterium Tuberculosis-Infected Macrophages Drives Anti- 
Inflammatory Responses and Inhibits Th1 Polarization of Responding T Cells. Infect Immun (2015) 83(6):2242-54. doi: 10.1128/IAI.00135-15

22. Long EM, Millen B, Kubes P, Robbins SM. Lipoteichoic Acid Induces Unique Inflammatory Responses When Compared to Other Toll-Like Receptor 2 Ligands. PLoS One (2009) 4(5):e5601. doi: 10.1371/journal.pone.0005601

23. Shim JH, Xiao C, Paschal AE, Bailey ST, Rao P, Hayden MS, et al. TAK1, But Not TAB1 or TAB2, Plays an Essential Role in Multiple Signaling Pathways In Vivo. Genes Dev (2005) 19(22):2668-81. doi: 10.1101/gad.1360605

24. Pulido R, Lang R. Dual Specificity Phosphatases: From Molecular Mechanisms to Biological Function. Int J Mol Sci (2019) 20(18):4372. doi: $10.3390 /$ ijms 20184372

25. Jeffrey KL, Camps M, Rommel C, Mackay CR. Targeting Dual-Specificity Phosphatases: Manipulating MAP Kinase Signalling and Immune Responses. Nat Rev Drug Discov (2007) 6(5):391-403. doi: 10.1038/nrd2289

26. Hammer M, Mages J, Dietrich H, Servatius A, Howells N, Cato AC, et al. Dual Specificity Phosphatase 1 (DUSP1) Regulates a Subset of LPS-induced Genes and Protects Mice From Lethal Endotoxin Shock. J Exp Med (2006) 203 (1):15-20. doi: 10.1084/jem.20051753

27. Zhao Q, Shepherd EG, Manson ME, Nelin LD, Sorokin A, Liu Y. The Role of Mitogen-Activated Protein Kinase Phosphatase-1 in the Response of Alveolar Macrophages to Lipopolysaccharide: Attenuation of Proinflammatory Cytokine Biosynthesis Via Feedback Control of P38. J Biol Chem (2005) 280(9):8101-8. doi: 10.1074/jbc.M411760200
28. Murray PJ. Understanding and Exploiting the Endogenous interleukin-10/ STAT3-mediated Anti-Inflammatory Response. Curr Opin Pharmacol (2006) 6(4):379-86. doi: 10.1016/j.coph.2006.01.010

29. Ananieva O, Darragh J, Johansen C, Carr JM, McIlrath J, Park JM, et al. The Kinases MSK1 and MSK2 Act as Negative Regulators of Toll-Like Receptor Signaling. Nat Immunol (2008) 9(9):1028-36. doi: 10.1038/ ni. 1644

30. Brenner S, Prosch S, Schenke-Layland K, Riese U, Gausmann U, Platzer C. Camp-Induced Interleukin-10 Promoter Activation Depends on CCAAT/ Enhancer-Binding Protein Expression and Monocytic Differentiation. J Biol Chem (2003) 278(8):5597-604. doi: 10.1074/jbc.M207448200

Conflict of Interest: The authors declare that the research was conducted in the absence of any commercial or financial relationships that could be construed as a potential conflict of interest.

Copyright $\odot 2021$ Francisco, Arranz, Merino, Punzón, Perona and Fresno. This is an open-access article distributed under the terms of the Creative Commons Attribution License (CC BY). The use, distribution or reproduction in other forums is permitted, provided the original author(s) and the copyright owner(s) are credited and that the original publication in this journal is cited, in accordance with accepted academic practice. No use, distribution or reproduction is permitted which does not comply with these terms. 